\title{
Livedo reticularis: a cutaneous clue to an underlying endocrine crisis
}

\author{
Natasha Shrikrishnapalasuriyar', Mirena Noyvirt', Philip Evans', Bethan Gibson², \\ Elin Foden² and Atul Kalhan \\ 'Departments of Diabetes and Endocrinology and Intensive Care, Royal Glamorgan Hospital, Llantrisant, UK
}

Correspondence should be addressed to $\mathrm{N}$ Shrikrishnapalasuriyar Email

natashashrikrishna@doctors. org.uk

\begin{abstract}
A 54-year-old woman was admitted to hospital with a presumed allergic reaction to a single dose of amoxicillin given for a suspected upper respiratory tract infection. She complained of chest tightness although there was no wheeze or stridor. On examination, she was pyrexial, tachycardic, hypertensive and had a diffuse mottled rash on her lower limbs. Her initial investigations showed raised inflammatory markers. She was treated in the intensive care for a presumed anaphylactic reaction with an underlying sepsis. Further investigations including CT head and CSF examination were unremarkable; however, a CT abdomen showed a $10 \mathrm{~cm}$ heterogeneous right adrenal mass. Based on review by the endocrine team, a diagnosis of pheochromocytoma crisis was made, which was subsequently confirmed on 24-h urinary metanephrine measurement. An emergency adrenalectomy was considered although she was deemed unfit for surgery. Despite intensive medical management, her conditioned deteriorated and she died secondary to multi-organ failure induced by pheochromocytoma crisis.
\end{abstract}

\section{Learning points:}

- Pheochromocytoma have relatively higher prevalence in autopsy series $(0.05-1 \%)$ suggestive of a diagnosis, which is often missed.

- Pheochromocytoma crisis is an endocrine emergency characterized by hemodynamic instability induced by surge of catecholamines often precipitated by trauma and medications ( $\beta$ blockers, general anesthetic agents, ephedrine and steroids).

- Pheochromocytoma crisis can mimic acute coronary syndrome, cardiogenic or septic shock.

- Livedo reticularis can be a rare although significant cutaneous marker of underlying pheochromocytoma crisis.

\section{Background}

Pheochromocytoma crisis (PMC) is characterized by hemodynamic instability induced by a surge of catecholamines, which can often be precipitated by medications (steroids, dopamine receptor antagonists, anticholinergics, general anesthetic agents etc.) or trauma (1). It can mimic other medical emergencies such as acute coronary syndrome, cardiogenic or septic shock. Livedo reticularis may be a rare although significant cutaneous clue to an underlying diagnosis of PMC. Management of PMC involves early use of $\alpha$-adrenergic blockers (prior to $\beta$-adrenergic blockers) in addition to close monitoring and hemodynamic supportive measures. The timing of adrenalectomy in such patients is debatable although based on our experience and a recently published European case series, an emergency adrenalectomy should be considered in patients presenting with life-threatening PMC. 


\section{Case report}

A 54-year-old woman was admitted to hospital with a presumed allergic reaction to a single dose of amoxicillin given $6 \mathrm{~h}$ earlier for a suspected upper respiratory tract infection. She was previously well and not on any regular medication. She had experienced transient episodes of turning pale and feeling lightheaded. These episodes were ongoing for 18 months prior to admission. Her partner described these as 'white outs' and family attributed these to her going through the menopause. The paramedic team had noticed a diffuse non-blanching rash on the lower half of her body, which had developed within a few hours after taking a single dose of amoxicillin from her primary care physician that day. Paramedics commenced intravenous adrenaline and hydrocortisone although her blood pressure was recorded to be $192 / 122 \mathrm{~mm}$ of $\mathrm{Hg}$, and there were no clinical signs of airways obstruction.

On admission, she complained of chest tightness although there was no wheeze or stridor. On examination, there was a diffuse mottled vasculitic rash (livedo reticularis) on lower half of her body and limbs (Figure 1). She was pyrexial, hypertensive and persistently tachycardic. Her chest and cardiovascular examination were unremarkable. Her baseline investigations were carried out including an ECG, which showed sinus tachycardia and chest $\mathrm{X}$ ray that was deemed normal. Her laboratory results showed an elevated white cell count, $\mathrm{C}$ reactive protein and deranged liver function as well as coagulation profile. She also had biochemical evidence of acute kidney injury (Table 1). Due to the

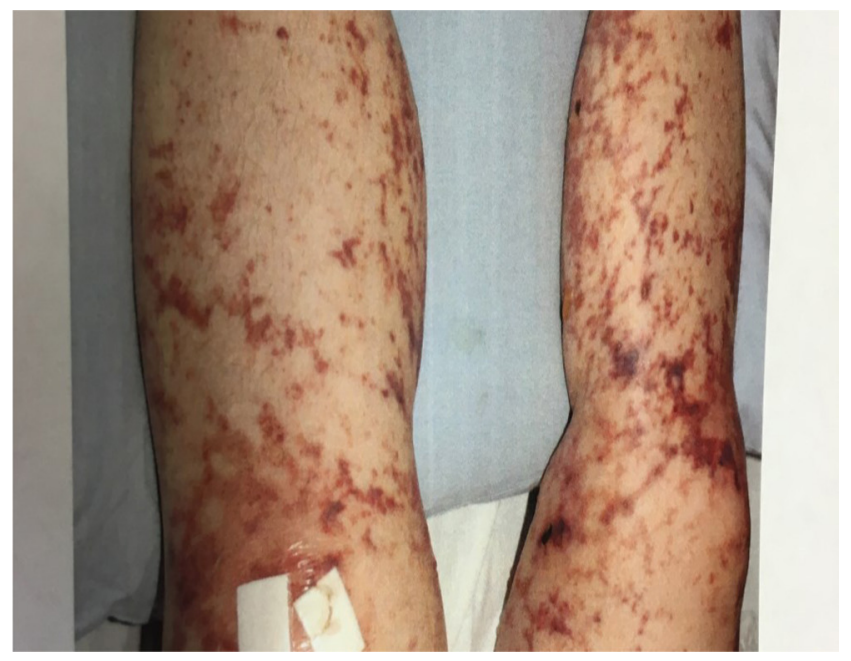

Figure 1

Livedo reticularis on presentation.
Table 1 Initial blood results.

\begin{tabular}{l} 
Investigation \\
\hline WCC \\
Neutrophils \\
Urea \\
Creatinine \\
CRP \\
Bilirubin \\
Albumin \\
ALP \\
ALT
\end{tabular}

\begin{tabular}{c}
\hline Result \\
\hline $36.1 \times 10^{9}$ \\
$34.1 \times 10^{9}$ \\
$22.6 \mathrm{mmol} / \mathrm{L}$ \\
$155 \mu \mathrm{mol} / \mathrm{L}$ \\
$116 \mathrm{mg} / \mathrm{L}$ \\
$55 \mu \mathrm{mol} / \mathrm{L}$ \\
$38 \mathrm{~g} / \mathrm{L}$ \\
$104 \mathrm{U} / \mathrm{L}$ \\
$5374 \mathrm{U} / \mathrm{L}$
\end{tabular}

\begin{tabular}{c}
\hline Normal value \\
\hline $4-11.0$ \\
$1.7-7.5$ \\
$2.5-7.8$ \\
$46-120$ \\
$<5$ \\
$<21$ \\
$35-50$ \\
$30-130$ \\
$<33$
\end{tabular}

acute onset of the rash and elevated inflammatory markers, she was treated for a presumed anaphylactic reaction to amoxicillin with an underlying systemic infection. A baseline septic screen was arranged. She was started on broad spectrum antibiotics (as per hospital protocol), intravenous fluids and supportive therapy in the intensive care unit.

On day 3 of admission, she became restless, agitated with an altered level of consciousness and clinical features suggestive of global encephalopathy. CT head, lumbar puncture and initial septic screen were unremarkable. Further radiological investigations including CT abdomen were carried out to identify a possible source of her ongoing sepsis.

\section{Investigations}

Table 1 shows initial abnormality in bloods, which led us to order a CT abdomen to find the underlying case of sepsis. Once the CT scan had been arranged (Figure 2), we then carried out 24 urinary metanephrines.

\section{Treatment}

Patient was admitted to the intensive care unit as she developed atrial fibrillation with fast ventricular rate with an associated drop in her blood pressure. She received noradrenaline to maintain a systolic blood pressure of $100 \mathrm{mmHg}$; however, her blood pressure remained labile, and after $72 \mathrm{~h}$, the noradrenaline was discontinued. CT abdomen, which was arranged to identify the possible source of sepsis, showed a $10 \mathrm{~cm}$ heterogeneous right adrenal mass (Figure 2). Following an urgent Endocrinology review, a possible diagnosis of pheochromocytoma crisis was considered, and she was given intravenous phentolamine. This diagnosis was subsequently confirmed on 24-h urinary metanephrine measurement (Table 2). Her blood pressure stabilized 


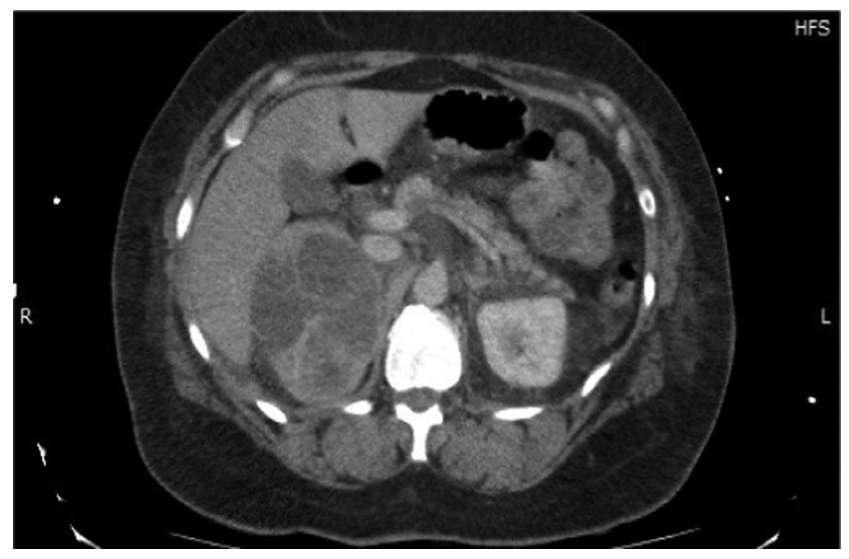

Figure 2

$10 \mathrm{~cm}$ heterogeneous right adrenal mass on CT.

within receiving $36 \mathrm{~h}$ of intravenous phentolamine therapy. After four days of intravenous treatment, she was switched to oral phenoxybenzamine given through nasogastric tube.

\section{Outcome and follow-up}

During her 10-day stay in intensive care, she had a cardiac arrest requiring $45 \mathrm{~min}$ of cardiopulmonary resuscitation (CPR). Post-CPR echocardiogram showed an ejection fraction of $40 \%$. An endocrine surgical opinion was requested for consideration of emergency adrenalectomy. This was deemed to be inappropriate as she had a multiorgan failure and her Glasgow Coma Scale (GCS) remained low despite intensive medical management. After 16 days of close monitoring and the best of medical efforts, she continued to deteriorate and died while in the intensive care unit.

\section{Discussion}

Pheochromocytoma and paragangliomas are rare endocrine tumors with incidence of $2-8$ per million persons per year. In patients with hypertension, the prevalence of these tumors range from 0.2 to $0.6 \%$ (2). About $5 \%$ of adrenal incidentalomas have been reported

Table 2 24-h urinary metanephrines/catecholamines.

\begin{tabular}{l}
\hline Investigation \\
\hline Normetadrenaline \\
Metadrenaline \\
Noradrenaline \\
Adrenaline \\
Dopamine \\
\hline
\end{tabular}

\begin{tabular}{c}
\hline Result \\
\hline $153.7 \mu \mathrm{mol}$ \\
$273.21 \mu \mathrm{mol}$ \\
$33,828 \mathrm{nmol}$ \\
$9088 \mathrm{nmol}$ \\
$6452 \mathrm{nmol}$ \\
\hline
\end{tabular}

Normal value $<4.40$ $<2.00$ $<900$ $<190$ $<4440$ to be associated with excess catecholamine secretion (3). Pheochromocytomas have a relatively higher prevalence in autopsy series $(0.05-0.1 \%)$ suggestive of a diagnosis, which is often under-recognized (2). Our patient had a history of episodic light-headedness and 'white outs' which were present for over a year prior to her admission. In hindsight, further evaluation of her pheochromocytomainduced paroxysmal episodes could have been life-saving.

Pheochromocytoma multisystem crisis (PMC) is characterized by hemodynamic instability (hyper and/ or hypotension), encephalopathy, fever $\left(>40^{\circ} \mathrm{C}\right)$ and multi-organ failure (1). It is induced by a sudden surge of catecholamines in the circulation, often precipitated by iatrogenic factors (metoclopramide, $\beta$ adrenergic blockers, ephedrine, general anesthetic agents and hydrocortisone), trauma or even manual handling. Our patient had been given a single dose of amoxicillin for a presumed upper respiratory tract infection, but the non-specific symptoms of feeling generally unwell may have been related to her massively increased catecholamine levels due to underlying pheochromocytoma. In view of her history and symptoms of breathlessness, tachycardia and rash, she received treatment for suspected anaphylaxis from the paramedics although she did not have any objective evidence of bronchospasm. It is likely that intravenous adrenaline and hydrocortisone precipitated the PMC.

Our patient also had livedo reticularis on admission, which remains an extremely rare cutaneous marker associated with pheochromocytoma (5). Livedo reticularis develops as a result of catecholamineinduced arteriolar vasospasm, which causes pooling of blood in the surrounding venules. There are only a few case reports in the medical literature describing pheochromocytoma presenting with livedo reticularis. The earliest case report published in 1974 describes a 32-year-old woman presenting with blotches in the skin, which on biopsy were suggestive of necrotizing vasculitis. She presented 6 months later with blood pressure of 210/150 and unaltered skin condition that prompted further investigations, which confirmed the diagnosis of pheochromcytoma (6). Buckley et al. (4) describe a 24-year-old man presenting with livedoid rash on arms, legs and body, which improved after right adrenalectomy. Histopathological analysis of adrenal mass was consistent with underlying diagnosis of pheochromocytoma (4).

PMC can mimic cardiogenic shock, acute coronary syndrome, sepsis of unknown origin and acute encephalitis. The clinical presentation varies greatly, and there are reports of PMC presenting with abdominal pain, anemia, back pain and acidosis (7). The diagnosis requires 
a high index of suspicion particularly as in our patient who was previously well and initial treatment was on lines of sepsis of unknown origin.

The timing of adrenalectomy in a patient with PMC is still to be defined. Kakioki et al. reviewed a case series of 13 patients with PMC in whom all 5 patients who were not operated died while the rest of the 8 patients who underwent emergency adrenalectomy survived (7). In our patient, despite aggressive management of hemodynamic instability, there was progressive deterioration, and she eventually died after close to 2 weeks of hospital stay. Our case further opens the debate regarding the exact timing for emergency adrenalectomy in such patients.

\section{Declaration of interest}

The authors declare that there is no conflict of interest that could be perceived as prejudicing the impartiality of the research reported.

\section{Funding}

This research did not receive any specific grant from any funding agency in the public, commercial or not-for-profit sector

\section{References}

1 Rosas AL, Kasperlik-Zaluska AA, Papierska L, Bass BL, Pacak K \& Eisenhofer G. Phaeochromocytoma crisis induced by glucocorticoids: a report of 4 cases and review of the literature. European Journal of Endocrinology 2008158 423-429. (https://doi.org/10.1530/EJE-07-0778)

2 Mansmann G, Lau J, Balk E, Rothberg M, Miyachi Y \& Bornstein SR. The clinically inapparent adrenal mass: update in diagnosis and management. Endocrine Reviews 200425 309-340. (https://doi. org/10.1210/er.2002-0031)

3 Fahmy WFH, Issa BG, Sim J, Keevil B \& Fryer A. Management of incidental adrenal tumours. British Medical Journal 2018360 J5674. (https://doi.org/10.1136/bmj.j5674)

4 Buckley SA, Lessing JN \& Mark NM.Livedo reticularis in a patient with phaeochromocytoma resolving after adrenalectomy. Journal of Clinical Endocrinology and Metabolism 201398 439-440. (https://doi. org/10.1210/jc.2012-2842)

5 Silburn M, Macmillan DC, Vickers HR Ledingham JG. Phaeochromocytoma with livedo reticularis. Proceedings Royal Society Medicine 197164 1193-1194.

6 Newell KA, Prinz RA, Pickleman J, Braithwaite S, Brooks M, Karson TH \& Glisson S. Phaeochromocytoma multisystem crisis. A surgical emergency. Archives of Surgery 1988123 956-959. (https:// doi.org/10.1001/archsurg.1988.01400320042007)

7 Kakoki K, Miyata Y, Shida Y, Hakariya T, Takehara K, Izumida S, Sekino M, Kinoshita N, Igawa T, Fukuoka J, et al. Phaeochromocytoma multisystem crisis treated with emergency surgery: a case report and literature review. BMC Research Notes 2015 8 758. (https://doi.org/10.1186/s13104-015-1738-z)

Received in final form 7 March 2018

Accepted 20 March 2018 